\title{
Comment on: local control and survival after induction chemotherapy and ablative radiation versus resection for pancreatic ductal adenocarcinoma with vascular involvement
}

\author{
Pauline Georges, Alexandre Doussot \\ Department of Digestive Surgical Oncology - Liver Transplantation Unit, CHU Besancon, Besancon, France \\ Correspondence to: Alexandre Doussot. Department of Digestive Surgical Oncology - Liver Transplantation Unit, CHU Besancon, 3, Boulevard \\ Fleming, 25000 Besancon, France. Email: adoussot@chu-besancon.fr. \\ Comment on: Jolissaint JS, Reyngold M, Bassmann J, et al. Local control and survival after induction chemotherapy and ablative radiation \\ versus resection for pancreatic ductal adenocarcinoma with vascular involvement. Ann Surg 2021. [Epub ahead of print]. doi: 10.1097/ \\ SLA.0000000000005080.
}

Submitted Aug 16, 2021. Accepted for publication Aug 27, 2021.

doi: 10.21037/hbsn-21-336

View this article at: https://dx.doi.org/10.21037/hbsn-21-336

We read with interest the study from Jolissaint $e t$ al. recently issued in the Annals of Surgery (1). In this single center retrospective study, the authors compared oncological outcomes in patients with pancreatic ductal adenocarcinoma (PDAC) and vascular involvement who underwent pancreatectomy $(\mathrm{n}=105) v s$. high dose ablative radiation (ART, $n=104)$. All patients received induction chemotherapy first, over a recent study period [2014-2018]. While the rationale for submitting patients to pancreatectomy or A-RT was not clearly stated in the Methods section, comparison between the two groups showed that patients allocated to A-RT were frailer with higher Charlson Comorbidity indexes and Eastern Cooperative Oncology Group (ECOG) scores and harbored significantly larger tumors, more often left-sided, with more arterial involvement as compared to patients in the pancreatectomy group. Notably, $70.2 \%$ of patients receiving A-RT were deemed locally advanced as compared to $19 \%$ in the pancreatectomy group. In other words, A-RT was delivered to patients ultimately deemed unresectable or unfit for resection. Regarding safety, major adverse event/morbidity and mortality rates in the A-RT and pancreatectomy groups were respectively $25 \% v s$. $28.6 \%$ and $4.8 \%$ vs. $2.9 \%$. From an oncological standpoint, despite no difference in terms of locoregional recurrence/ progression between A-RT and pancreatectomy groups at 18 months $(16 \%$ vs. $21 \%, \mathrm{P}=0.252)$, patients in the A-RT group experienced significantly more distant recurrence/ progression $(58 \%$ vs. $30 \%, \mathrm{P}=0.004)$. This difference translated in a 12.8-month median overall survival (OS) difference $(\mathrm{P}<0.001)$ with a significantly worse 2 -year OS rate in the A-RT group ( $38 \%$ vs. $62 \%, \mathrm{P}<0.001$ ).

Overall, the authors should be acknowledged as they address a trending topic and present somehow thoughtprovoking results. Indeed, pancreatectomy with vascular resection, especially arterial, is associated with significant morbidity and mortality rates and remains debated in terms of survival benefit $(2,3)$. In this setting, alternative local therapies are appealing, especially for patients initially or ultimately deemed unresectable or unfit for resection.

Yet, the current study deserves cautious interpretation as several points must be considered. First, as discussed by the authors, head-to-head comparison between the two groups remains inadequate due to obvious selection bias. A-RT was indeed allocated to frailer patients with more advanced disease than patients who underwent pancreatectomy. Most patients who received A-RT had locally advanced disease whereas most in the pancreatectomy group had borderline resectable disease. Consequently, this difference might suggest a more aggressive tumor biology in the A-RT group leading to less favorable outcomes. Nevertheless, pancreatectomy being the cornerstone of PDAC treatment, this difference may also be imputed to the absence of resection.

Second, inherently to its retrospective design, management may have differed between groups. For instance, all patients in both groups received induction 
chemotherapy, either FOLFIRINOX/mFOLFIRINOX or gemcitabine and nab-paclitaxel. However, comparative data on the number of cycles, doses or tolerance are lacking. One can wonder whether patients in the A-RT group being frailer with more comorbidity received comparable treatment as compared to patients eventually resected. Similarly, management varied also among groups. In the pancreatectomy group, nearly one-third of patients $(\mathrm{n}=32)$ received additional chemoradiotherapy (CRT) after induction chemotherapy. CRT following induction chemotherapy has been associated with improved local control for borderline and locally advanced PDAC whereas its beneficial impact on survival remains unclear (4-7). Yet, beyond its potential impact on survival, adding CRT implies a time-lead bias in survival analysis of the pancreatectomy group, the so-called immortal time bias (8). In the Methods section, the authors defined survival as calculated from the completion date of induction chemotherapy to the date of last follow-up or death to account for immortal time bias inherent to induction chemotherapy. Immortal time refers to a period of follow-up during which, due to study design, the outcome of interest cannot occur. In essence, patients in the pancreatectomy group receiving additional CRT after induction chemotherapy could not die or develop disease progression during CRT, otherwise they would have been ineligible for surgery and consequently not included in the pancreatectomy group. Duration of CRT should be also considered as immortal time to avoid survival overestimation. It is unclear whether the authors accounted for this bias in the subset of patients operated on after additional CRT. Nevertheless, the authors performed a sensitivity analysis excluding those patients who received additional CRT before pancreatectomy $(n=32)$ and confirmed longer OS in the pancreatectomy group with a 14.9-month median OS difference $(\mathrm{P}<0.001)$ as compared to the A-RT group.

Finally, as highlighted by the differences between the groups due to treatment allocation bias, comparing A-RT to resection might be inappropriate. As aforementioned, because complete tumor removal constitutes the most effective local control, complete resection stands as the only curative intent management whenever achievable, in selected patients. Thus, the difference in terms of survival favoring the pancreatectomy group may be imputed to the absence of resection in the A-RT group. Instead of comparing outcomes after resection vs. A-RT, comparing A-RT to additional conventional CRT in a prospective setting would be more informative on the value of A-RT.
In conclusion, this study adds to the growing body of literature suggesting a role for radiation in the management of borderline and locally advanced PDAC (9-11). A-RT stands as a potential approach for delivering definitive radiation to achieve durable local control in patients with locally unresectable PDAC or deemed medically inoperable. Still, as confirmed in the authors study (R0 rate $=61.2 \%$ ), complete R0 resection is challenging to achieve in case of borderline and locally advanced disease. In this setting, A-RT may help increasing local control before pancreatectomy thereby facilitating $\mathrm{R} 0$ resection. Induction chemotherapy followed by A-RT before pancreatectomy could represent a future strategy to provide durable systemic and local disease control. In the present study, two patients underwent pancreatectomy after A-RT and three had aborted resection after A-RT. Both resectability and surgical safety after A-RT will be awaited data from future prospective investigations.

\section{Acknowledgments}

Funding: None.

\section{Footnote}

Provenance and Peer Review: This article was commissioned by the editorial office of Hepatobiliary Surgery and Nutrition. The article did not undergo external peer review.

Conflicts of Interest: Both authors have completed the ICMJE uniform disclosure form (available at https://hbsn. amegroups.com/article/view/10.21037/hbsn-21-336/coif). The authors have no conflicts of interest to declare.

Ethical Statement: The authors are accountable for all aspects of the work in ensuring that questions related to the accuracy or integrity of any part of the work are appropriately investigated and resolved.

Open Access Statement: This is an Open Access article distributed in accordance with the Creative Commons Attribution-NonCommercial-NoDerivs 4.0 International License (CC BY-NC-ND 4.0), which permits the noncommercial replication and distribution of the article with the strict proviso that no changes or edits are made and the original work is properly cited (including links to both the formal publication through the relevant DOI and the license). See: https://creativecommons.org/licenses/by-nc-nd/4.0/. 


\section{References}

1. Jolissaint JS, Reyngold M, Bassmann J, et al. Local control and survival after induction chemotherapy and ablative radiation versus resection for pancreatic ductal adenocarcinoma with vascular involvement. Ann Surg 2021. [Epub ahead of print]. doi: 10.1097/ SLA.0000000000005080.

2. Raptis DA, Sánchez-Velázquez P, Machairas $\mathrm{N}$, et al. Defining benchmark outcomes for pancreatoduodenectomy with portomesenteric venous resection. Ann Surg 2020;272:731-7.

3. Bachellier P, Addeo P, Faitot F, et al. Pancreatectomy with arterial resection for pancreatic adenocarcinoma: how can it be done safely and with which outcomes?: a single institution's experience with 118 patients. Ann Surg 2020;271:932-40.

4. Pietrasz D, Turrini O, Vendrely V, et al. How does chemoradiotherapy following induction FOLFIRINOX improve the results in resected borderline or locally advanced pancreatic adenocarcinoma? An AGEOFRENCH multicentric cohort. Ann Surg Oncol 2019;26:109-17..

5. Murphy JE, Wo JY, Ryan DP, et al. Total neoadjuvant therapy with FOLFIRINOX followed by individualized chemoradiotherapy for borderline resectable pancreatic adenocarcinoma: a phase 2 clinical trial. JAMA Oncol 2018;4:963-9. Erratum in: JAMA Oncol 2018;4:1439.

6. Hammel P, Huguet F, van Laethem JL, et al. Effect of

Cite this article as: Georges $\mathrm{P}$, Doussot A. Comment on: local control and survival after induction chemotherapy and ablative radiation versus resection for pancreatic ductal adenocarcinoma with vascular involvement. HepatoBiliary Surg Nutr 2021;10(5):672-674. doi: 10.21037/hbsn-21-336 chemoradiotherapy vs chemotherapy on survival in patients with locally advanced pancreatic cancer controlled after 4 months of gemcitabine with or without erlotinib: the LAP07 randomized clinical trial. JAMA 2016;315:1844-53.

7. Janssen QP, van Dam JL, Kivits IG, et al. Added value of radiotherapy following neoadjuvant FOLFIRINOX for resectable and borderline resectable pancreatic cancer: a systematic review and meta-analysis. Ann Surg Oncol 2021. [Epub ahead of print]. doi: 10.1245/s10434-02110276-8.

8. Lévesque LE, Hanley JA, Kezouh A, et al. Problem of immortal time bias in cohort studies: example using statins for preventing progression of diabetes. BMJ 2010;340:b5087.

9. Goldsmith C, Plowman PN, Green MM, et al. Stereotactic ablative radiotherapy (SABR) as primary, adjuvant, consolidation and re-treatment option in pancreatic cancer: scope for dose escalation and lessons for toxicity. Radiat Oncol 2018;13:204.

10. Harrison JM, Wo JY, Ferrone CR, et al. Intraoperative radiation therapy (IORT) for borderline resectable and locally advanced pancreatic ductal adenocarcinoma (BR/ LA PDAC) in the era of modern neoadjuvant treatment: short-term and long-term outcomes. Ann Surg Oncol 2020;27:1400-6.

11. Reyngold M, O'Reilly EM, Varghese AM, et al. Association of ablative radiation therapy with survival among patients with inoperable pancreatic cancer. JAMA Oncol 2021;7:735-8. 